\title{
Evaluation of basis sets with 11-electron analytic effective core potentials of gold for modeling molecular electronic devices
}

\author{
Shimin Hou*, Rui Li, Zekan Qian, Jiaxing Zhang, Ziyong Shen, Xingyu Zhao, Zenquan Xue
}

Key Laboratory for the Physics and Chemistry of Nanodevices, Department of Electronics, Peking University, Beijing 100871, China

The corresponding author. Email: $\underline{\text { smhou@ } @ \text { ku.edu.cn }}$

Here we give additional information regarding the influence of different exchange-correlation (XC) functionals on DFT calculation results of a neutral Au atom in ground state, the Au-BDT-Au complex and the 1D monatomic gold chain. Two generalized gradient approximation (GGA) functionals (BPW91 and PBEPBE) and two hybrid functions (B3LYP and B3PW91) are considered [1-5].

Table 1 shows Kohn-Sham eigenenergies of a single $\mathrm{Au}$ atom in ground state calculated in spinunrestricted formalism using BPW91, PBEPBE, B3LYP and B3PW91 functionals. The same conclusion as that of using the BLYP functional can be drawn, that is, both the TMDZ and TMSZ basis sets give eigenenergies comparable to those of the SDD basis, while LANL1DZ, LANL1MB and ECMB all underbind the s-state and overbind d-states heavily.

In Table 2 are listed the Au-S bond length, atomic charge on Au atoms and binding energy of the optimized Au-BDT-Au complex calculated using BPW91, PBEPBE, B3LYP and B3PW91 functionals, and Table 3 gives orbital character analysis of the Au-S bond using these functionals. In like manner, the performance of TMDZ and TMSZ is much superior to that of LANL1DZ, LANL1MB and ECMB although they are developed using the specific BLYP functional.

In Fig.1 are given the band structure and density of states (DOS) of the 1D gold monatomic chain 
calculated using the TMSZ basis combined with BLYP, BPW91, PBEPBE, B3LYP and B3PW91 functionals. It can be seen that all these functionals give almost the same correct result, except for some minor details. However, the TMDZ basis combined with BPW91, PBEPBE, B3LYP and B3PW91 functionals cannot deliver correct band structure and DOS of the gold chain. Hence, the TMDZ basis set is recommended for geometry optimization and the TMSZ basis set is recommended for electronic structure and electronic transport calculations.

Reference

(1) Becke A.D. Phys. Rev. A, 1988, 38,3098

(2) Perdew J.P.; Wang Y. Phys.Rev.B, 1992, 45, 13244

(3) Perdew J.P.; Burke K.; Ernzerhof M. Phys. Rev. Lett., 1996, 77, 3865

(4) Becke A.D. J.Chem.Phys., 1993, 89, 5648

(5) Lee C.; Yang W.; Parr R.G. Phys.Rev. B, 1988, 37, 785 
Table 1 Comparison of Kohn-Sham eigenenergies of a single Au atom in ground state

\begin{tabular}{|c|c|c|c|c|}
\hline & & $\varepsilon_{5 \mathrm{~d} \uparrow}(\mathrm{eV})$ & $\varepsilon_{5 \mathrm{~d} \downarrow}(\mathrm{eV})$ & $\varepsilon_{6 \mathrm{~s} \uparrow}(\mathrm{eV})$ \\
\hline \multirow{7}{*}{ BPW91 } & LANL2DZ & -7.31 & -7.05 & -6.22 \\
\hline & SDD & -7.07 & -6.79 & -6.07 \\
\hline & TMDZ & -7.01 & -6.71 & -6.15 \\
\hline & TMSZ & -7.24 & -6.90 & -6.22 \\
\hline & ECMB & -10.49 & -10.19 & -3.45 \\
\hline & LANL1DZ & -9.06 & -8.81 & -5.00 \\
\hline & LANL1MB & -10.55 & -10.25 & -5.47 \\
\hline \multirow[t]{7}{*}{ PBEPBE } & LANL2DZ & -7.28 & -7.02 & -6.18 \\
\hline & SDD & -7.08 & -6.79 & -6.05 \\
\hline & TMDZ & -7.03 & -6.72 & -6.15 \\
\hline & TMSZ & -7.15 & -6.81 & -6.17 \\
\hline & ECMB & -10.40 & -10.09 & -3.41 \\
\hline & LANL1DZ & -9.03 & -8.77 & -4.97 \\
\hline & LANL1MB & -10.46 & -10.16 & -5.43 \\
\hline \multirow[t]{7}{*}{ B3LYP } & LANL2DZ & -8.52 & -8.30 & -6.73 \\
\hline & SDD & -8.33 & -8.09 & -6.62 \\
\hline & TMDZ & -7.94 & -7.64 & -6.87 \\
\hline & TMSZ & -7.80 & -7.46 & -6.80 \\
\hline & ECMB & -11.07 & -10.77 & -4.02 \\
\hline & LANL1DZ & -9.98 & -9.73 & -5.67 \\
\hline & LANL1MB & -11.13 & -10.84 & -6.04 \\
\hline \multirow[t]{7}{*}{ B3PW91 } & LANL2DZ & -8.57 & -8.32 & -6.77 \\
\hline & SDD & -8.35 & -8.08 & -6.73 \\
\hline & TMDZ & -7.95 & -7.62 & -6.96 \\
\hline & TMSZ & -7.92 & -7.54 & -6.93 \\
\hline & ECMB & -11.19 & -10.85 & -4.12 \\
\hline & LANL1DZ & -10.02 & $-9.75 \mathrm{e}$ & -5.74 \\
\hline & LANL1MB & -11.24 & -10.91 & -6.13 \\
\hline
\end{tabular}


Table 2 Comparison of Au-S bond lengths, atomic charge on $\mathrm{Au}$ atoms and binding energies of the optimized Au-BDT-Au complex

\begin{tabular}{|c|c|c|c|c|}
\hline & & bond length $(\AA)$ & atomic charge & $\mathrm{BE}(\mathrm{Ev})$ \\
\hline \multirow{7}{*}{ BPW91 } & LANL2DZ & 2.33 & 0.21 & 2.90 \\
\hline & SDD & 2.31 & 0.19 & 3.08 \\
\hline & TMDZ & 2.34 & 0.19 & 2.72 \\
\hline & TMSZ & 2.34 & 0.18 & 2.54 \\
\hline & ECMB & 2.38 & 0.44 & 1.93 \\
\hline & LANL1DZ & 2.42 & 0.37 & 2.08 \\
\hline & LANL1MB & 2.44 & 0.29 & 1.77 \\
\hline \multirow[t]{7}{*}{ PBEPBE } & LANL2DZ & 2.32 & 0.20 & 3.19 \\
\hline & SDD & 2.32 & 0.19 & 3.35 \\
\hline & TMDZ & 2.34 & 0.19 & 3.00 \\
\hline & TMSZ & 2.34 & 0.18 & 2.82 \\
\hline & ECMB & 2.37 & 0.44 & 2.17 \\
\hline & LANL1DZ & 2.41 & 0.36 & 2.35 \\
\hline & LANL1MB & 2.44 & 0.29 & 2.01 \\
\hline \multirow[t]{7}{*}{ B3LYP } & LANL2DZ & 2.34 & 0.24 & 2.69 \\
\hline & SDD & 2.33 & 0.22 & 2.84 \\
\hline & TMDZ & 2.32 & 0.20 & 2.77 \\
\hline & TMSZ & 2.33 & 0.19 & 2.61 \\
\hline & ECMB & 2.41 & 0.47 & 1.85 \\
\hline & LANL1DZ & 2.42 & 0.38 & 2.12 \\
\hline & LANL1MB & 2.44 & 0.31 & 1.72 \\
\hline \multirow[t]{7}{*}{ B3PW91 } & LANL2DZ & 2.32 & 0.26 & 2.93 \\
\hline & SDD & 2.31 & 0.24 & 3.09 \\
\hline & TMDZ & 2.32 & 0.21 & 3.05 \\
\hline & TMSZ & 2.32 & 0.20 & 2.86 \\
\hline & ECMB & 2.36 & 0.49 & 2.04 \\
\hline & LANL1DZ & 2.40 & 0.41 & 2.30 \\
\hline & LANL1MB & 2.42 & 0.33 & 1.87 \\
\hline
\end{tabular}


Table 3 Comparison of orbital character of the Au-S bond in the optimized Au-BDT-Au complex

\begin{tabular}{|c|c|c|c|c|c|c|c|}
\hline & \multicolumn{2}{|c|}{$\%$ s character } & \multicolumn{2}{|c|}{$\%$ p character } & \multicolumn{2}{|c|}{$\%$ d character } \\
\hline & & $\mathrm{Au}$ & $\mathrm{S}$ & $\mathrm{Au}$ & $\mathrm{S}$ & $\mathrm{Au}$ & $\mathrm{S}$ \\
\hline \multirow[t]{7}{*}{ BPW91 } & LANL2DZ & 87.50 & 3.94 & 0.19 & 95.83 & 12.31 & 0.22 \\
\hline & SDD & 87.20 & 3.74 & 0.30 & 96.03 & 12.50 & 0.23 \\
\hline & TMDZ & 88.04 & 3.40 & 0.20 & 96.43 & 11.76 & 0.17 \\
\hline & TMSZ & 89.12 & 3.14 & 0.30 & 96.70 & 10.59 & 0.17 \\
\hline & ECMB & 93.04 & 2.22 & 5.66 & 97.59 & 1.30 & 0.19 \\
\hline & LANL1DZ & 97.50 & 2.13 & 0.17 & 97.78 & 2.33 & 0.10 \\
\hline & LANL1MB & 97.72 & 1.82 & 0.55 & 98.09 & 1.73 & 0.10 \\
\hline \multirow[t]{7}{*}{ PBEPBE } & LANL2DZ & 87.17 & 4.05 & 0.20 & 95.72 & 12.63 & 0.23 \\
\hline & SDD & 86.92 & 3.79 & 0.30 & 95.97 & 12.78 & 0.24 \\
\hline & TMDZ & 87.80 & 3.50 & 0.20 & 96.32 & 12.00 & 0.18 \\
\hline & TMSZ & 88.89 & 3.22 & 0.27 & 96.61 & 10.84 & 0.17 \\
\hline & ECMB & 93.04 & 2.20 & 5.60 & 97.60 & 1.36 & 0.20 \\
\hline & LANL1DZ & 97.38 & 2.15 & 0.16 & 97.75 & 2.46 & 0.10 \\
\hline & LANL1MB & 97.66 & 1.85 & 0.51 & 98.05 & 1.83 & 0.10 \\
\hline \multirow[t]{7}{*}{ B3LYP } & LANL2DZ & 88.49 & 4.22 & 0.21 & 95.54 & 11.30 & 0.24 \\
\hline & SDD & 88.41 & 4.04 & 0.35 & 95.72 & 11.23 & 0.25 \\
\hline & TMDZ & 85.88 & 4.04 & 0.23 & 95.74 & 13.88 & 0.22 \\
\hline & TMSZ & 86.78 & 3.79 & 0.29 & 96.00 & 12.94 & 0.21 \\
\hline & ECMB & 92.01 & 2.67 & 6.51 & 97.13 & 1.48 & 0.21 \\
\hline & LANL1DZ & 97.05 & 2.55 & 0.22 & 97.33 & 2.73 & 0.12 \\
\hline & LANL1MB & 97.27 & 2.19 & 0.60 & 97.69 & 2.13 & 0.11 \\
\hline \multirow[t]{7}{*}{ B3PW91 } & LANL2DZ & 88.69 & 4.57 & 0.24 & 95.19 & 11.07 & 0.25 \\
\hline & SDD & 88.54 & 4.30 & 0.39 & 95.44 & 11.08 & 0.26 \\
\hline & TMDZ & 85.72 & 4.28 & 0.29 & 95.48 & 13.99 & 0.24 \\
\hline & TMSZ & 86.51 & 4.06 & 0.39 & 95.72 & 13.10 & 0.23 \\
\hline & ECMB & 91.35 & 2.66 & 7.10 & 97.11 & 1.55 & 0.23 \\
\hline & LANL1DZ & 96.97 & 2.79 & 0.30 & 97.09 & 2.74 & 0.12 \\
\hline & LANL1MB & 96.97 & 2.40 & 0.82 & 97.48 & 2.21 & 0.12 \\
\hline
\end{tabular}




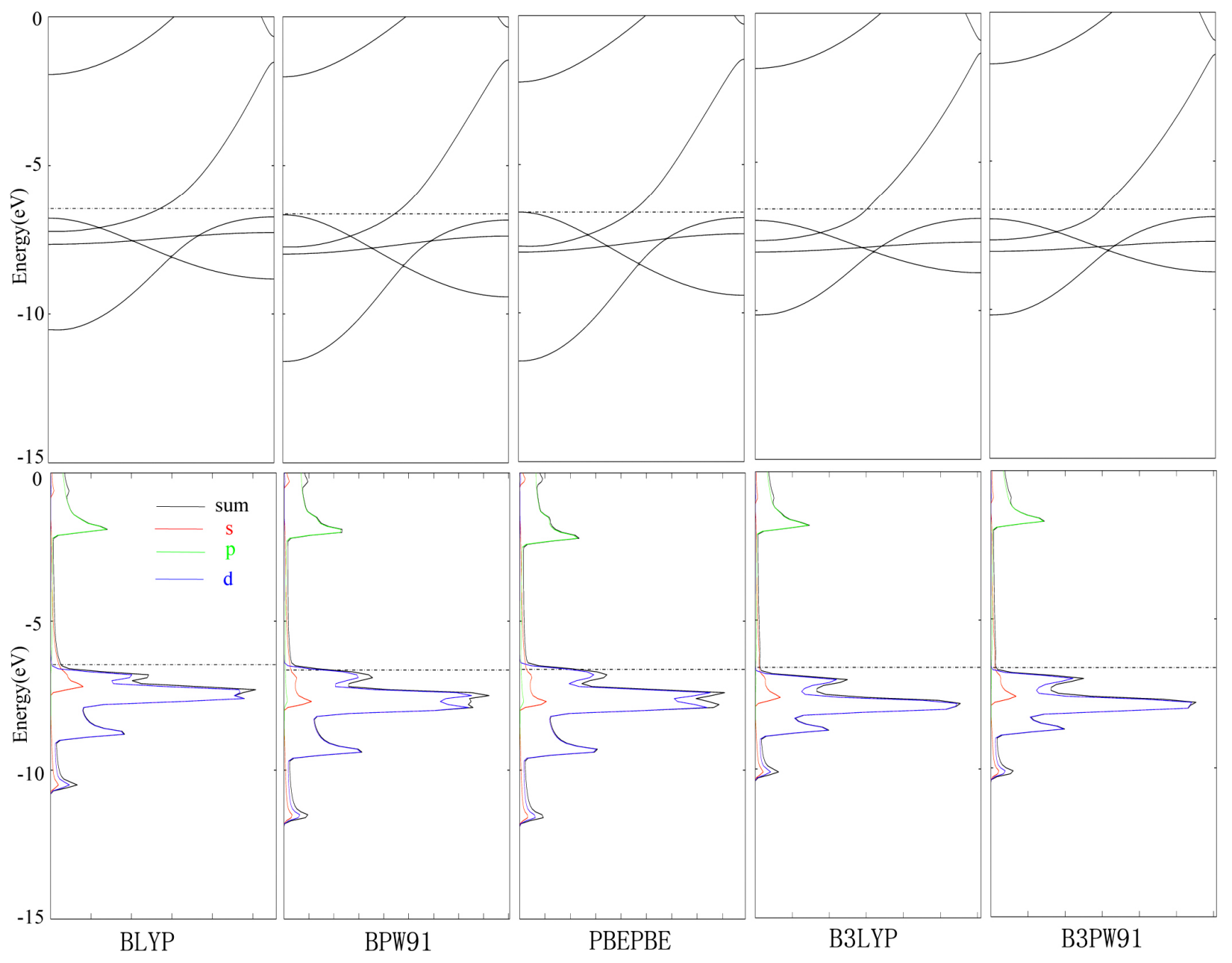

Figure 1 Band structures and DOS of an infinite 1D monatomic chain of gold 